\title{
Refining the role of de novo protein truncating variants in neurodevelopmental disorders using population reference samples
}

Jack A. Kosmicki ${ }^{1-5}$, Kaitlin E. Samocha ${ }^{1-3,5}$, Daniel P. Howrigan ${ }^{1-3}$, Stephan J. Sanders ${ }^{6}$, Kamil Slowikowski ${ }^{2,4,7,8}$, Monkol Lek ${ }^{1,2}$, Konrad J. Karczewski ${ }^{1,2}$, David J. Cutler ${ }^{9}$, Bernie Devlin ${ }^{10}$, Kathryn Roeder $^{11}$, Joseph D. Buxbaum ${ }^{12-17}$, Benjamin M. Neale ${ }^{1-3}$, Daniel G. MacArthur ${ }^{1,2}$, Dennis P. Wall ${ }^{18}$, Elise B. Robinson ${ }^{1-3}$, Mark J. Daly ${ }^{1-3}$

${ }^{1}$ Analytic and Translational Genetics Unit, Department of Medicine, Massachusetts General Hospital and Harvard Medical School, Boston, MA 02114, USA

${ }^{2}$ Program in Medical and Population Genetics, Broad Institute of Harvard and MIT, 7 Cambridge Center, Cambridge, MA 02142, USA

${ }^{3}$ Stanley Center for Psychiatric Research, Broad Institute of Harvard and MIT, Cambridge, MA 02142, USA

${ }^{4}$ Program in Bioinformatics and Integrative Genomics, Harvard University, Cambridge, MA 02138, USA

${ }^{5}$ Program in Genetics and Genomics, Biological and Biomedical Sciences, Harvard Medical School, Boston, MA 02446, USA

${ }^{6}$ Department of Psychiatry, University of California, San Francisco, San Francisco, California 94158, USA

${ }^{7}$ Divisions of Genetics and Rheumatology, Department of Medicine, Brigham and Women's Hospital and Harvard Medical School, Boston, MA 02446, USA

${ }^{8}$ Partners Center for Personalized Genetic Medicine, Boston, MA 02446, USA

${ }^{9}$ Department of Human Genetics, Emory University School of Medicine, Atlanta, GA 30322, USA

${ }^{10}$ Department of Psychiatry, University of Pittsburgh School of Medicine, Pittsburgh, PA 15213, USA

${ }^{11}$ Department of Statistics, Carnegie Mellon University, Pittsburgh, PA 15213, USA

${ }^{12}$ Seaver Autism Center for Research and Treatment, Icahn School of Medicine at Mount Sinai, New York, NY 10029, USA

${ }^{13}$ Department of Psychiatry, Icahn School of Medicine at Mount Sinai, New York, NY 10029, USA

${ }^{14}$ Department of Neuroscience, Icahn School of Medicine at Mount Sinai, New York, NY 10029, USA

${ }^{15}$ Friedman Brain Institute, Icahn School of Medicine at Mount Sinai, New York, NY 10029, USA

${ }^{16}$ Department of Genetics and Genomic Sciences, Icahn School of Medicine at Mount Sinai, New York, NY 10029, USA

${ }^{17}$ Mindich Child Health and Development Institute, Icahn School of Medicine at Mount Sinai, New York, NY 10029, USA

${ }^{18}$ Departments of Pediatrics (Systems Medicine), Biomedical Data Science, and Psychiatry (by courtesy), Stanford University, CA, 94305, USA

\section{Address for Correspondence:}


bioRxiv preprint doi: httos://doi org/101101/052886: this version posted Februarv 8,2017 The copvriaht holder for this preprint (which was not certified by peer review) is the author/funder, who has granted bioRxiv a license to display the preprint in perpetuity. It is made available under aCC-BY-ND 4.0 International license.

\section{Mark J. Daly}

Chief, Analytic and Translational Genetics Unit Massachusetts General Hospital

617-643-3290

mjdaly@,atgu.mgh.harvard.edu 


\begin{abstract}
Recent research has uncovered an important role for de novo variation in neurodevelopmental disorders. Using aggregated data from 9246 families with autism spectrum disorder, intellectual disability, or developmental delay, we show $\sim 1 / 3$ of de novo variants are independently observed as standing variation in the Exome Aggregation Consortium's cohort of 60,706 adults, and these de novo variants do not contribute to neurodevelopmental risk. We further use a loss-of-function (LoF)-intolerance metric, pLI, to identify a subset of LoF-intolerant genes that contain the observed signal of associated de novo protein truncating variants (PTVs) in neurodevelopmental disorders. LoF-intolerant genes also carry a modest excess of inherited PTVs; though the strongest de novo impacted genes contribute little to this, suggesting the excess of inherited risk resides lower-penetrant genes. These findings illustrate the importance of population-based reference cohorts for the interpretation of candidate pathogenic variants, even for analyses of complex diseases and de novo variation.
\end{abstract}

\title{
Introduction
}

Autism spectrum disorders (ASDs) are a phenotypically heterogeneous group of heritable disorders that affect $\sim 1$ in 68 individuals in the United States ${ }^{1}$. While estimates of the common variant (heritable) contribution toward ASD liability are upwards of $50 \%{ }^{2-4}$, few specific risk variants have been identified, in part because ASD GWAS sample sizes to date remain limited. Conversely, the field made substantial progress understanding the genetic etiology of ASD via analysis of de novo (newly arising) variation using exome sequencing of parent-offspring trios ${ }^{5-}$ 10. Severe intellectual disability and developmental delay (ID/DD) are considerably less heritable than ASDs ${ }^{11}$ (though frequently comorbid) and have demonstrated a stronger contribution from de novo frameshift, splice acceptor, splice donor, and nonsense variants (collectively termed protein truncating variants [PTVs] ${ }^{12-14}$. Furthermore, ASD cases with comorbid ID show a significantly higher rate of de novo PTVs than those with normal or above average $\mathrm{IQ}^{6,9,15-17}$, while higher IQ cases have a stronger family history of neuropsychiatric disease $^{15}$, suggesting a greater heritable contribution.

De novo variants comprise a unique component of the genetic architecture of human disease since, having not yet passed through a single generation, any heterozygous variants with complete or near-complete elimination of reproductive fitness must reside almost exclusively in this category. Despite prior evidence of mutational recurrence ${ }^{18}$ (i.e., the same mutation occurring de novo in multiple individuals), most studies implicitly assumed each de novo variant was novel, in line with Kimura's infinite sites model ${ }^{19}$, and thereafter analyzed de novo variants genome-wide without respect to their allele frequency in the population (Supplementary Note). However, the mutation rate is not uniform across the genome, with some regions and sites experiencing higher mutation rates than others (e.g., $\mathrm{CpG}$ sites ${ }^{20}$ ). A classic example comes from achondroplasia, in which the same G-to-C and G-to-T variant at a $\mathrm{CpG}$ site was observed de novo in 150 and 3 families, respectively ${ }^{18}$. As such, it should not be surprising to observe a de novo variant at a given site and also observe the same variant (defined as one with the same chromosome, position, reference, and alternate allele) present as standing variation in the population.

Given the strong selective pressure on neurodevelopmental disorders ${ }^{21-23}$, we expect most highly deleterious (high-risk conferring) de novo PTVs will linger in the population for at most a few 
generations. Thus, the collective frequency of such variants in the population will approximate their mutation rate. Individual PTVs tolerated to be seen in relatively healthy adults, and more generally PTVs in genes that tolerate the survival of such variants in the population, may be less likely to contribute significant risk to such phenotypes, and are therefore permitted by natural selection to reach allele frequencies orders of magnitude larger than those of highly deleterious variants. Given the current size of the human population ( $\sim 7$ billion), and the expectation of one de novo variant per exome ( 1 in $\sim 30$ million bases), every non-embryonic lethal coding mutation is likely present as a de novo variant at least once in the human population. This reasoning, along with the availability of large exome sequencing reference databases, motivated our interest in searching for variants observed de novo in trio sequencing studies that are also present as standing variation in the human population, indicating a recurrent mutation. We will herein refer to these de novo variants that are also observed as standing variation in the population as class 2 de novo variants, with the remaining de novo variants referred to as class 1 de novo variants (i.e., observed only de novo; Fig. 1).

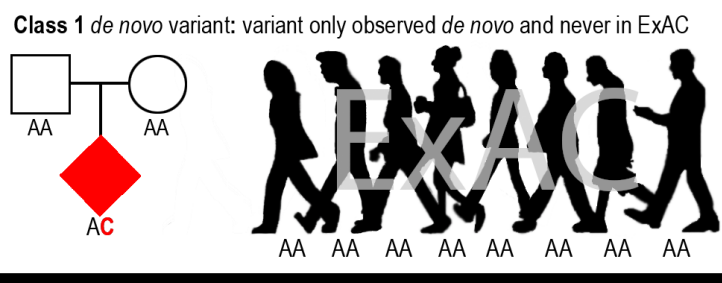

Class 2 de novo variant: variant observed both de novo \& as standing variation in ExAC
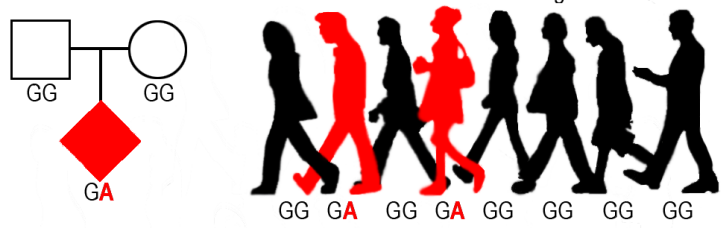

Figure 1 Illustration of class 1 and class 2 de novo variants with the genotypes of each variant for 8 of the 60,706 individuals in ExAC.

With the release of the Exome Aggregation Consortium's (ExAC) dataset of 60,706 adult individuals without severe developmental abnormalities $^{24}$, we can now empirically investigate the rate and relative pathogenicity of recurrent mutations. While there have been many studies examining de novo variation in human disease, the success in ASD and ID/DD, coupled with the large sample sizes published to date, led us to focus our evaluation on these phenotypes.

\section{Results}

\section{Class 2 de novo variation}

We first asked how many of the 10,093 variants observed de novo in ID/DD cases ${ }^{13}$, ASD cases, and unaffected ASD siblings are also observed as standing variation in the 60,706 reference exomes from $\operatorname{ExAC}^{24}$ (Fig. 1; Online Methods). We found that 1854 ASD (31.66\%), 841 unaffected ASD sibling (33.05\%), and $410 \mathrm{ID} / \mathrm{DD}$ (24.23\%) de novo variants were observed as standing variation in one or more ExAC individuals (class 2 de novo variants) (Fig. 2A; Supplementary Tables 1-3). When we removed the 15,330 exomes originating from psychiatric cohorts (many of which are controls), the rate of class 2 de novo variation drops to $28.47 \%( \pm 1.03 \%, 95 \% \mathrm{CI})$, a rate statistically indistinguishable from the expected recurrence rate of $28.13 \%( \pm 0.42 \%, 95 \%$ CI; two-sided binomial test; $P=0.45$; Fig. 2B; Supplementary Figs. 1 \& 2; Supplementary Table 4; Online Methods). We found similar rates of class 2 de novo variants in published trio studies of schizophrenia ${ }^{25}$ and congenital heart disease ${ }^{26,27}$

(Supplementary Tables $5 \&$ 6). While the presence of class 2 de novo variants is not a novel observation $^{18,25}$, the rate is approximately three times larger than previous estimates ${ }^{25}$ owing to significantly larger reference datasets (Fig. 2B; Supplementary Fig. 2). 
We first sought confirmation that the observed recurrence rate - the proportion of variants observed both de novo and as standing variation in the population - was technically sound and not the result of some undetected contamination or missed heterozygote calls in parents (i.e., false de novo variants). Five secondary analyses strongly support the technical validity of this work. 1) In line with previous publications ${ }^{25}$, class 2 de novo variants, regardless of their functional impact, are enriched at $\mathrm{CpG}$ sites as compared to class 1 de novo variants $\left(P<1 \times 10^{-}\right.$ ${ }^{20}$; Fisher's exact test; Supplementary Table 7). 2) As the exomes used to call de novo variants in De Rubeis et al. (2014) were used in the joint calling of ExAC, and many were sequenced at the same center as the majority of ExAC samples, it is possible that false class 2 de novo variants could be elevated in this dataset via contamination or joint calling artifacts. However, we observe no difference in the rate of class 2 de novo variation between De Rubeis et al. (2014) and Iossifov, O'Roak, Sanders, Ronemus et al. (2014) $(P=0.10$; Fisher's exact test; Supplementary Table 8). 3) The frequency distribution of class 2 de novo variants should be skewed dramatically upward towards common variation if contamination or missed parental heterozygotes were contributing; however, the ExAC frequency of class 2 de novo variants at $\mathrm{CpGs}$ were indistinguishable from all such variants in ExAC compared to variants drawn at random with the same annotation and $\mathrm{CpG}$ content $(P=0.14$; Wilcoxon rank sum test; Fig. 2C). 4) In fact, a subset of synonymous variants experimentally validated in the ASD studies showed nearly the same recurrence rate as the overall set, most definitively establishing that these mutations indeed arose independently ( $P=0.60$; Fisher's exact test). 5) Lastly, it is well documented that mutation rate increases with paternal age ${ }^{28-31}$, thus rates of both class 1 and class 2 de novo variants should show association with paternal age if both classes were genuine de novo variants. Indeed, for the 1861 unaffected ASD siblings with available paternal age information, rates of both class 1 and class 2 de novo variants are associated with increasing paternal age (class $1: \beta=0.002, P=4.11 \times 10^{-9}$; class $2: \beta=0.0009, P=1.06 \times 10^{-5}$; linear regression).

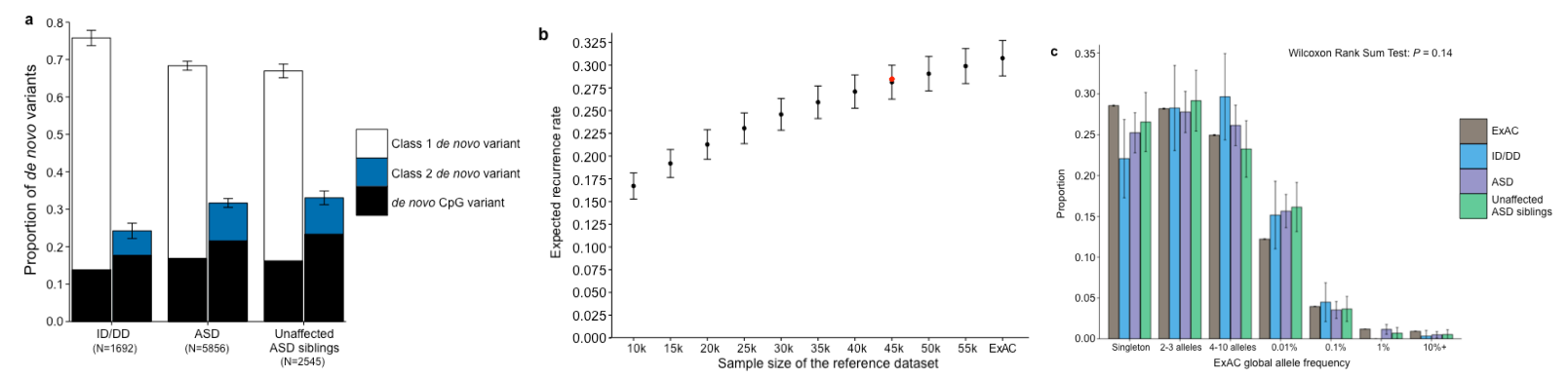

Figure 2 Properties of class 2 de novo variants. (a) The proportion of de novo variants across each cohort split between class 1 (left) and class 2 (right) with $\mathrm{CpG}$ variants marked in black. Class 2 de novo variants are strongly enriched for $\mathrm{CpG}$ variants $\left(P<10^{-20}\right)$. The corresponding figure using the non-psychiatric version of ExAC can be found in Supplementary Figure 2. (b) Expected recurrence rate (rate of class 2 de novo variants across ID/DD, ASD, and unaffected ASD siblings) given the sample size of the reference dataset. The red dot indicates the observed recurrence rate of the non-psychiatric version of ExAC. (c) Allele frequency distribution of class 2 de novo $\mathrm{CpGs}$ by cohort with the matching distribution of $\mathrm{CpG}$ variants in ExAC for comparison. Allele frequency distributions do not significantly differ $(P=0.14$; Wilcoxon rank sum test $)$. Error bars represent $95 \%$ confidence intervals throughout (a) - (c). ID/DD, intellectual disability / developmental delay; ASD, autism spectrum disorder

We then sought to determine whether class 1 and class 2 de novo variants contribute equally to ASD and ID/DD risk. As a control for the comparison of functional variants, rates of both class 
1 and class 2 de novo synonymous variants are equivalent across ASD, ID/DD, and unaffected ASD siblings (Fig. 3A; Supplementary Table 9) and remain unchanged when we removed the psychiatric cohorts within ExAC (Supplementary Fig. 3A; Supplementary Table 10). Thus, collectively neither class 1 nor class 2 de novo synonymous variants demonstrated an association with ASD or ID/DD, consistent with previous reports that as a class, de novo synonymous variants do not contribute to risk $\mathrm{k}^{5-10}$. While previous reports implicated de novo PTVs as significant risk factors for $\mathrm{ASD}^{5,6,15,16}$ and ID/DD ${ }^{13}$, the class 2 de novo subset of PTVs show no such association for either ASD ( 0.015 per case vs. 0.023 per unaffected ASD sibling; $P=0.98$; one-sided Poisson exact test $\left.{ }^{32}\right)$ or ID/DD ( 0.016 per case vs. 0.023 per unaffected ASD sibling; $P=0.94$; one-sided Poisson exact test), with slightly higher rates in unaffected ASD siblings (Fig. 3B; Supplementary Table 11). By contrast, after removing class 2 de novo PTVs, class 1 de novo PTVs are significantly more enriched in individuals with ASD (0.13 per case) and ID/DD (0.19 per case) as opposed to unaffected ASD siblings (0.07 per control) (ASD vs. control: rate ratio $[\mathrm{RR}]=1.83 ; P=6.07 \times 10^{-12}, \mathrm{ID} / \mathrm{DD}$ vs. control: $\mathrm{RR}=2.61 ; P=6.31 \times 10^{-21}$; one-sided Poisson exact test). The lack of excess case burden in class 2 de novo variants was consistent with what would be expected if such variants did not contribute to ASD and ID/DD risk. However, to ensure we were not losing causal variants by removing all de novo variants found in ExAC, we tested the class 2 de novo PTVs at three ExAC allele frequency (AF) thresholds: singletons (1 allele in ExAC), AF $<0.0001$, and $\mathrm{AF}<0.001$. We found no significant difference between the rate of class 2 de novo PTVs between individuals with ID/DD or ASD as compared to unaffected ASD siblings at any threshold (Fig. 3C; Supplementary Table 12). Furthermore, these results remain consistent regardless of whether the psychiatric exomes in ExAC are retained or excluded, demonstrating they are not driving the observed associations (Supplementary Fig. 3B; Supplementary Table 13). Thus, the data provides no evidence to suggest these class 2 de novo variants contribute to the previously observed enrichment of de novo variation in ASD and ID/DD cases, and removing those variants present in ExAC increases the effect size for de novo PTVs in ASD and ID/DD. Moving forward, we focus our analyses solely on variation absent from ExAC.

\section{Gene level analyses}

Since observed risk to ASD or ID/DD was carried only by de novo variants absent from the standing variation of ExAC, we next sought to extend this concept by evaluating whether the overall rate of PTVs per gene in ExAC provided a similar guide to which ASD and ID/DD variants were relevant. Specifically, we investigated whether the gene-level constraint metric, $\mathrm{pLI}^{16}$ (probability of loss-of-function intolerance), could improve our ability to decipher which class 1 de novo PTVs confer the most risk to ASD and ID/DD (Online Methods). Using the same threshold as Lek et al. (2016), we used a threshold of $\mathrm{pLI} \geq 0.9$ to define loss-of-function (LoF)-intolerant genes and investigated whether individuals with ASD had an increased burden of class 1 de novo PTVs in such genes. When we restricted to solely class 1 de novo PTVs in LoF-intolerant genes, we observed a significant excess in individuals with ASD $(0.067$ per exome) compared to their unaffected siblings $\left(0.021\right.$ per exome; $\mathrm{RR}=3.24 ; P=3.14 \times 10^{-16}$; onesided Poisson exact test). For individuals with ID/DD, the rate of class 1 de novo PTVs in LoFintolerant genes becomes more striking, with a rate of 0.139 per exome, resulting in a $6.70 \mathrm{RR}$ when compared to the control group of unaffected ASD siblings $\left(P=6.34 \times 10^{-38}\right.$; one-sided Poisson exact test). By contrast, the rate of class 1 de novo PTVs in LoF-tolerant genes (pLI $<0.9$ ) show no difference between individuals with ASD (0.063 vs. $0.051 ; P=0.06$; two-sided 
Poisson test), or individuals with ID/DD ( 0.048 vs. $0.051 ; P=0.75$; two-sided Poisson exact test; Fig. 3D; Supplementary Table 14) when compared to unaffected ASD siblings. Again, results remain unchanged when we exclude the ExAC psychiatric samples (Supplementary Fig. 3C; Supplementary Table 15). The same trend is observed in congenital heart disease ${ }^{26,27}$ and schizophrenia $^{25}$ (Supplementary Note; Supplementary Tables 16-21). Hence, all detectable de novo PTV signal in these phenotypes can be localized to $18 \%$ of genes with clear intolerance to PTVs in ExAC, with, consequently, substantially amplified rate ratios in this gene set.
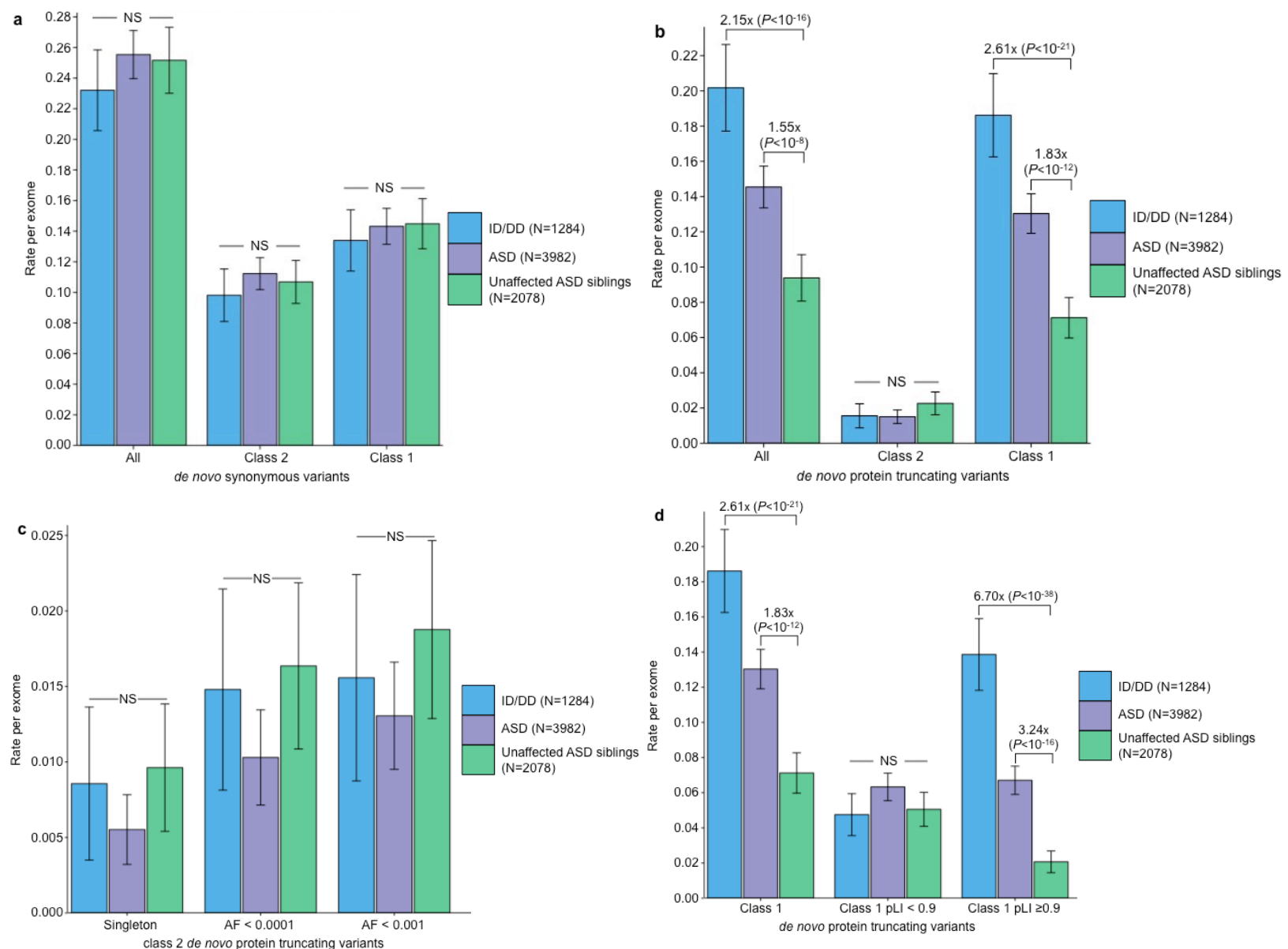

Figure 3 Partitioning the rate of de novo variants per exome by class 1, class 2, and pLI. Within each grouping, the rate - variants per individual - is shown for ID/DD (left), ASD (middle), and unaffected ASD siblings (right) with the number of individuals labeled in the legends. (a) Rate of de novo synonymous variants per exome partitioned into class 2 (middle) and class 1 (right). No significant difference was observed for any grouping of de novo synonymous variants. (b) Rate of de novo PTVs per exome partitioned into class 2 (middle) and class 1 (right). Only class 1 de novo PTVs in ID/DD and ASD show association when compared to unaffected ASD siblings. (c) Rate of class 2 de novo PTVs broken by different ExAC global allele frequency (AF) thresholds: singleton (observed once; left), AF $<0.0001$ (middle), and AF $<0.001$ (right). (d) Rate of class 1 de novo PTVs partitioned into class 1 de novo PTVs in $\mathrm{pLI} \geq 0.9$ genes (right), and class 1 de novo PTVs in $\mathrm{pLI}<0.9$ genes (middle). The entire observed association for de novo PTVs resides in class 1 de novo PTVs in pLI $\geq 0.9$ genes. For all such analyses, the rate ratio and significance were calculated by comparing the rate for ID/DD and ASD to the rate in unaffected ASD siblings using a two-sided Poisson exact test ${ }^{32}$ for synonymous variants and one-sided for the remainder (Online Methods). Error bars represent 95\% confidence intervals throughout (a) - (d). See Supplementary Fig. 3 for the corresponding figures using the non-psychiatric version of ExAC. ID/DD, intellectual disability / developmental delay; ASD, autism spectrum disorder; PTV, protein truncating variant; pLI, probability of loss-of-function intolerance; NS, not significant 
Recent studies inferred the presence of multiple de novo PTVs in the same gene as evidence of contribution to ASD risk ${ }^{5-10}$. Of the 51 genes with $\geq 2$ de novo PTVs, only 38 are absent in controls (Supplementary Table 22). This not only reinforces the point that the mere observation of multiple de novo PTVs in a gene is not sufficient to define that gene as important ${ }^{5,16}$, but also provides an opportunity to explore whether the pLI metric can refine the identification of specific genes. In fact, 32 of the 38 case-only genes, but only 5 of 13 controlonly or case-control hit genes, are LoF-intolerant, a highly significant difference $(\mathrm{OR}=8.07$; $P=0.003$; Fisher's exact test) that greatly refines the list of genes to be pursued as likely ASD contributors.

\section{Phenotypic associations for class 1 de novo PTVs in LoF-intolerant genes.}

While enrichment of de novo PTVs is one of the hallmarks of ASD de novo studies ${ }^{5-10,15,16}$, another consistent finding is an increased burden of these variants among females with ASD $^{6,15}$ and in ASD individuals with low full-scale IQ (FSIQ) ${ }^{6,15,16}$. We investigated whether these hallmarks were present in the $6.55 \%$ of ASD cases with a class 1 de novo PTV in LoF-intolerant genes ( $\mathrm{pLI} \geq 0.9)$. Indeed, females are overrepresented in the subset $(12.26 \%$ of females; $5.80 \%$ males; $P=1.75 \times 10^{-5}$; Fisher's exact test; Supplementary Table 23). Importantly, for the $6.86 \%$ of ASD cases with a class 2 de novo PTV or a class 1 de novo PTV in a LoF-tolerant gene (pLI $<0.9$ ), there is no difference between the sexes, with $6.86 \%$ of females and $6.83 \%$ of males falling in this category ( $P=1$; Fisher's exact test; Supplementary Table 24). Furthermore, class 2 de novo PTVs and class 1 de novo PTVs in LoF-tolerant genes show no association with FSIQ $(\beta=-0.001 ; P=0.76$; Poisson regression), while class 1 de novo PTVs in LoF-intolerant genes predominately explain the skewing towards lower FSIQ $\left(\beta=-0.023 ; P=7 \times 10^{-8}\right.$; Poisson regression; Fig. 4A). Given these observations, we split the ASD class 1 de novo PTV signal in LoF-intolerant genes by sex and intellectual disability status (Online Methods). Females with comorbid ASD and intellectual disability have the highest rate of class 1 de novo PTVs in LoFintolerant genes $\left(\mathrm{RR}=8.71 ; P=2.73 \times 10^{-12}\right.$; one-sided Poisson exact test $)$. Despite the overwhelming enrichment in females and individuals with comorbid ASD and intellectual disability, males with ASD without intellectual disability still show enrichment of class 1 de novo PTVs in LoF-intolerant genes (RR=2.95; $P=1.31 \times 10^{-9}$; one-sided Poisson exact test; Fig. 4B; Supplementary Table 25). These secondary analyses strongly support the implication of the primary analysis: that collectively, class 2 de novo PTVs and class 1 de novo PTVs in LoFtolerant genes have little to no association to ASD or ID/DD and no observable phenotypic impact on those cases carrying them. By contrast, the class 1 de novo variants occurring in LoFintolerant genes contain the association signal and phenotypic skewing observed to date. 

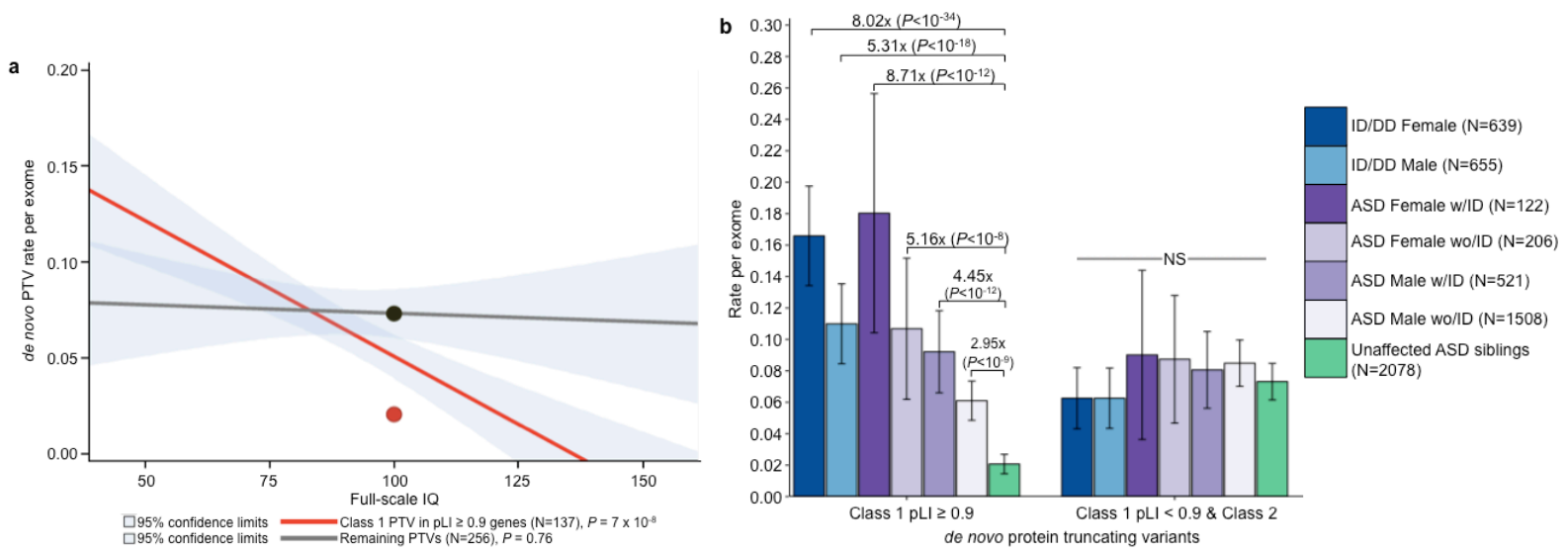

Figure 4 Phenotypic associations for ASD de novo PTVs. (a) IQ distribution of class 1 de novo PTVs in pLI $\geq 0.9$ genes (red) and remaining de novo PTVs (class 2 and class 1 pLI $<0.9$; grey) in 393 individuals with ASD with a measured full-scale IQ. Dots indicate the rate in unaffected ASD siblings for their respective categories of de novo PTVs. $P$-values calculated using Poisson regression. Only class 1 de novo PTVs show association with full-scale IQ. (b) Rate of class 1 de novo PTVs (left set) and the remaining de novo PTVs (class 2 \& class 1 in LoF-tolerant genes, right set) in ID/DD (left two bars) and ASD (middle four bars) split by sex and ID with the number of individuals labeled in the legends. Error bars represent $95 \%$ confidence intervals, and $P$-values were calculated using one-side Poisson exact tests comparing to unaffected ASD siblings. ASD, autism spectrum disorder; PTV, protein truncating variant; $\mathrm{pLI}$, probability of loss-of-function intolerance

\section{Inherited variation}

As the effect size for de novo PTVs increased after removing those variants present in ExAC, we postulated a similar increase could be obtained from rare inherited PTVs. Under the assumption that risk-conferring variants should be transmitted more often to individuals with ASD, we tested for transmission disequilibrium in a cohort of 4319 trios with an affected proband (Online Methods). Without filtering by pLI or presence/absence status in ExAC, singleton PTVs, as a class, showed no over-transmission $(P=0.31$; two-sided binomial test $)$. After removing all of the variants present in ExAC or in a LoF-tolerant gene $(\mathrm{pLI}<0.9)$, we found a modest excess of transmitted PTVs in ASD cases $\left(\mathrm{RR}=1.16 ; P=9.85 \times 10^{-3}\right.$; two-sided binomial test; Supplementary Table 26). As with all previous analyses, this result is virtually identical when the psychiatric cohorts in ExAC are removed ( $\mathrm{RR}=1.14 ; P=0.02$; two-sided binomial test). While there are far more inherited PTVs than de novo PTVs, the inherited variant effect size (1.16 RR) is paradoxically minute by comparison to that of de novo PTVs (3.24 RR).

Despite the different effect sizes between de novo and inherited PTVs, the data does not suggest the two classes of variation differ in penetrance. Instead, the data suggest the excess of inherited PTVs resides in a different set of genes than those implicated by de novo variation. Specifically, the largest de novo variant excess resides in a limited and extremely penetrant set of genes that do not contribute substantially to inherited PTV counts. If we consider the 11 genes with $\geq 3$ class 1 de novo PTVs in ASD cases and none in controls (47 de novo PTVs in total), all 11 are intolerant of truncating variation ( $\mathrm{pLI} \geq 0.9$ ) (Table 1; Supplementary Table 22). These variants confer risk to particularly severe outcomes: of the cases with available IQ data, 14 of the 29 individuals have IQ below 70 or were unable to complete a traditional IQ test ${ }^{15}$, while only $27 \%$ of all ASD individuals with available IQ data in this study fall into this group $(P=0.008$; Fisher's exact test). Across this same gene set, there are only 4 inherited PTVs (from a total of 5 observed in the parents of the 4,319 ASD trios). Of those 4 inherited PTVs, only the inherited 
frameshift in $C H D 8$ bore evidence of mosaic transmission $\left(P=5.49 \times 10^{-3}\right.$; two-sided binomial test; Supplementary Table 27) suggesting it may have arisen post-zygotically and not carried by a parent. This ratio - that $80-90 \%$ of the observed variants are de novo rather than inherited in ASD cases - indicates enormous selective pressure against mutations in these genes, far greater than the direct selection against ASD in general (Table 1). Indeed, despite ascertaining these 11 genes based on those with the most class 1 de novo PTVs in ASD, we observe a higher rate of de novo PTVs in these same genes in the ID/DD studies (37 mutations in 1284 cases). This underscores that selection against these variants likely arises from more severe and widespread impact on neurodevelopment and cognition. Despite the minor contribution of inherited variation in these genes, some insights from studying families may be particularly useful. To our surprise, 1 of the 4 inherited PTVs, a nonsense variant in $A N K 2$, was also observed de novo in an unrelated individual with ASD, providing a rare instance in which the same variant was observed both inherited and de novo in two unrelated individuals with ASD, yet absent from 60,706 individuals in ExAC (Supplementary Note).

Table 1 Top 12 genes with $\geq 3$ class 1 de novo PTVs in individuals with ASD

\begin{tabular}{lccccccccc}
\hline & \multicolumn{3}{c}{$\begin{array}{c}\text { Class 1 de novo PTVs } \\
\text { Unaffected }\end{array}$} & \multicolumn{7}{c}{ Inherited } & \multicolumn{2}{c}{ Case-control } & \\
Gene & ASD & ASD siblings & ID/DD & T & U & Case & Control & pLI & $P$-value \\
\hline CHD8 & 7 & 0 & 0 & 1 & 0 & 0 & 0 & 1 & $3.70 \mathrm{E}-13$ \\
ARID1B & 5 & 0 & 11 & 0 & 0 & 0 & 0 & 1 & $1.07 \mathrm{E}-08$ \\
DYRK1A & 5 & 0 & 2 & 0 & 0 & 0 & 0 & 0.9996 & $2.46 \mathrm{E}-11$ \\
SYNGAP1 & 5 & 0 & 9 & 0 & 0 & 0 & 0 & 1 & $2.47 \mathrm{E}-10$ \\
ADNP & 4 & 0 & 4 & 0 & 0 & 1 & 0 & 0.9989 & $3.93 \mathrm{E}-09$ \\
ANK2 & 4 & 0 & 0 & 1 & 1 & 0 & 0 & 1 & $7.07 \mathrm{E}-06$ \\
DSCAM & 4 & 0 & 0 & 2 & 0 & 1 & 0 & 1 & $3.62 \mathrm{E}-07$ \\
SCN2A & 4 & 0 & 7 & 0 & 0 & 1 & 0 & 1 & $1.25 \mathrm{E}-06$ \\
ASH1L & 3 & 0 & 0 & 0 & 0 & 0 & 0 & 1 & $1.67 \mathrm{E}-04$ \\
CHD2 & 3 & 0 & 2 & 0 & 0 & 0 & 0 & 1 & $7.81 \mathrm{E}-05$ \\
KDM5B & 3 & 2 & 0 & 5 & 1 & 0 & 0 & $5.09 \mathrm{E}-05$ & $7.22 \mathrm{E}-05$ \\
POGZ & 3 & 0 & 2 & 0 & 0 & 2 & 0 & 1 & $3.12 \mathrm{E}-05$ \\
\hline
\end{tabular}

12 genes with $\geq 3$ class 1 de novo PTVs in 3982 individuals with ASD. Additionally, for each gene, we have listed the number of class 1 de novo PTVs in 2078 unaffected ASD siblings and in 1284 individuals with ID/DD, as well as the number of singleton, LOFTEE (see URLs) high-confidence PTVs absent from ExAC that were transmitted (T) or untransmitted (U) to 4319 individuals with ASD and present in 404 cases of ASD and 3654 population controls. $P$-values represent the Poisson probability of observing more than the expected number of class 1 de novo PTVs (Online Methods). ID/DD, intellectual disability / developmental delay; ASD, autism spectrum disorder; PTV, protein truncating variant; $\mathrm{pLI}$, probability of loss-of-function intolerance

\section{Case-control analysis}

Having observed a significant enrichment in both de novo and inherited PTVs absent from ExAC in LoF-intolerant genes ( $\mathrm{pLI} \geq 0.9$ ), we applied this same methodology to case-control cohorts. Given that the variation present in a single individual will be a combination of de novo (both somatic and germline) and inherited variation, we expect to see an effect size for PTVs intermediate between that of the de novo and inherited PTVs absent from ExAC in LoFintolerant genes. Using a published cohort of 404 ASD cases and 3654 controls from Sweden ${ }^{5}$, 
we first analyzed the rate of singleton synonymous variants as a control for further analyses. We found no case-control difference among those present/absent from $\operatorname{ExAC}(P=0.59$; Fisher's exact test; Supplementary Table 28). Turning to the PTV category, we observe a slight excess of singleton PTVs in cases with ASD (917 PTVs in 404 cases) compared to controls (7259 PTVs in 3654 controls; $\mathrm{OR}=1.16 ; P=3.13 \times 10^{-5}$; Fisher's exact test; Supplementary Table 29). This signal increases once we remove all singleton PTVs present in ExAC or in LoF-tolerant genes, providing the first instance of an exome-wide excess of PTVs demonstrated in ASD without the use of trios (128 PTVs in 404 cases, 447 PTVs in 3654 controls; 2.63 OR; $P=1.37 \times 10^{-18}$; Fisher's exact test; Supplementary Table 30 \& 31). Consistent with the previous de novo and inherited analyses, no signal exists for the remaining 7601 singleton PTVs (OR=1.06; $P=0.11$; Fisher's exact test; Supplementary Table 32). Lastly, removing the psychiatric cohorts from ExAC results in a 2.42 OR for singleton PTVs absent from ExAC in LoF-intolerant genes (133 PTVs in 404 cases, 506 PTVs in 3654 controls; $P=1.06 \times 10^{-16}$; Fisher's exact test; Supplementary Table 33).

\section{Discussion}

Here we demonstrated that $\sim 1 / 3$ of de novo variants identified in neurodevelopmental disease cohorts are also present as standing variation in ExAC, indicating the presence of widespread mutational recurrence. Reinforcing this, we demonstrated that these class 2 de novo variants are enriched for more mutable $\mathrm{CpG}$ sites. Most importantly, however, these class 2 de novo variants confer no detectable risk to ID/DD and ASDs, and eliminating them from our analysis improved all genetic and phenotypic associations by removing the "noise" of benign variation.

We further refined the class 1 de novo PTV association using a gene-level intolerance metric (pLI) developed using the ExAC resource and identified that all detectable mutational excess resided in $18 \%$ of genes most strongly and recognizably intolerant of truncating mutation. Specifically, $13.5 \%( \pm 2.0 \%, 95 \% \mathrm{CI})$ of individuals with ID/DD and $6.55 \%( \pm 0.8 \%, 95 \% \mathrm{CI})$ of individuals with ASD, but only $2.1 \%( \pm 0.6 \%, 95 \% \mathrm{CI})$ of controls, have a de novo PTV absent from ExAC and present in a gene with a very low burden of PTVs in ExAC (pLI $\geq 0.9)$. ASD cases with such a variant are more likely to be female and/or have intellectual disability than the overall ASD population. For the remaining $93.45 \%$ of the ASD cohort, we fail to observe any meaningful phenotypic difference (i.e., IQ or sex) between the $6.86 \%$ of individuals with and the $86.59 \%$ of individuals without a class 2 de novo PTV or a class 1 de novo PTV in a LoF-tolerant gene. These results, taken together with an overall lack of excess case burden, suggest that collectively, neither class 2 nor class 1 de novo PTVs in LoF-tolerant genes ( $\mathrm{pLI}<0.9)$ appear to confer significant risk toward ASD. Thus, we have refined the role of de novo protein truncating variation in ASD, confining the signal to a smaller subset of patients than previously described $^{6,33}$.

This analysis framework, operating at the variant level, also enabled a careful examination of inherited variation in ASD. While ASD is highly heritable ${ }^{3}$, few analyses ${ }^{34}$ have demonstrated specific inherited components. By removing inherited PTVs present in ExAC or in LoF-tolerant genes, we discovered a modest signal of over-transmitted PTVs, in line with previous reports ${ }^{34}$. The vast majority of inherited PTVs appear to affect genes that have yet to show signal from de novo variation, with only $1 \%$ residing in the strongest associated genes, indicating the inherited variants reside in genes with a somewhat weaker selective pressure against them. Ultimately, 
however, as these variants occur in $15.4 \%$ of cases but carry only a 1.16 -fold increased risk as a group, they explain little of the overall heritability ( $<1 \%$ of the variance in liability).

Given the current size of ExAC and the general scarcity of truncating variants, the pLI metric for constraint against loss-of-function variation does not yet provide precise resolution of the selection coefficient acting on PTVs in that gene. That is, even a $\mathrm{pLI} \geq 0.9$ does not guarantee a selection coefficient sufficiently high to ensure the vast majority of variation is de novo rather than inherited. In fact, selection coefficients for $\mathrm{pLI} \geq 0.9$ genes range from $0.1-0.5$ (where the majority of variation will be inherited), all the way to selection coefficients approaching 1 , in which the variants are almost completely reproductively null. Only larger reference panels will enable refining these estimates, articulating a gradient from the strongest genes we currently flag (e.g., the 11 genes with $\geq 3$ de novo PTVs in ASD and none in controls that make their contribution almost entirely through penetrant, single-generation de novo variation) to those genes we have yet to define clearly that will make their contribution largely through inherited, albeit less penetrant, variation. The significant expansion of exome sequencing in ASD, alongside larger reference panels from which to draw more precise inferences about selective pressure against variation in each gene, will allow us to fill in the genetic architecture of ASDs in the region of the effect size spectrum between severe de novo variation at one end and common variation at the other.

ExAC currently has 15,330 individuals from psychiatric cohorts, with the schizophrenia cohort being the largest ${ }^{24}$. Given the shared genetics between ASD and schizophrenia ${ }^{2,5,16,17,25,35,36}$, it is reasonable to hypothesize that the psychiatric cohorts within ExAC could influence our analyses. As we have shown however, removing the psychiatric cohorts within ExAC does not change our results. In fact, of the 16 de novo PTVs in LoF-intolerant genes that were also variant in ExAC, only two reside solely in the 15,330 individuals from the psychiatric cohorts (CUX2 in ASD, $L A R P 1$ in unaffected ASD siblings). This number being so small is in retrospect not surprising because it is so unusual to observe a deleterious variant both de novo and present as standing variation in individuals with the same ascertained phenotype, let alone in different ascertained phenotypes. The $A N K 2$ nonsense variant was the only such instance of the same deleterious variant being de novo in one ASD trio and inherited in another.

While we use ASDs and ID/DD here to explore this framework, it can certainly be applied toward any trait. However, this framework is optimally powered in traits governed by genes under strong selection, as it will remove de novo variants that are more common when examined in the context of a larger reference population. Our results reinforce the point that not all de novo variants are rare and contribute to risk, while highlighting the tremendous value of large population sequence resources even for the interpretation of de novo variation and complex disease. This is especially important in the case of clinical sequencing, in which the paradigm has unfortunately become that if a protein-altering de novo variant is present in the gene of interest, then it is often considered the causal variant ${ }^{37,38}$. Clearly, not all de novo variants are equal, and not all de novo variants in a gene contribute to risk in the same way.

\section{Online Methods \\ Datasets and data processing.}


Two versions of the Exome Aggregation Consortium (ExAC) database were used in this analysis: the full version of $\operatorname{ExAC}(\mathrm{N}=60,706)$ and the non-psychiatric version of ExAC $(\mathrm{N}=$ 45,376). The non-psychiatric version of ExAC has the following cohorts removed: Bulgarian trios $(\mathrm{N}=461)$, sequencing in Suomi $(\mathrm{N}=948)$, Swedish schizophrenia \& bipolar studies $(\mathrm{N}=$ $12,119)$, schizophrenia trios from Taiwan $(\mathrm{N}=1505)$, and Tourette syndrome association international consortium for genomics $(\mathrm{N}=297)$. We used a combined set of 8401 published de novo variants from 3982 probands with ASD and 2078 of their unaffected siblings from two recent large-scale exome sequencing studies: de Rubeis et al $\left(\mathrm{N}_{\mathrm{ASD}}=1474, \mathrm{~N}_{\text {unaffected sib }}=267\right)^{5}$, Iossifov, O'Roak, Sanders, Ronemus et al $\left(\mathrm{N}_{\mathrm{ASD}}=2508, \mathrm{~N}_{\text {unaffected sib }}=1911\right)^{6}$ (Supplementary Table 1). We also used 1692 de novo variants from 1284 probands published in studies of intellectual disability (ID) (de Ligt et al: $\mathrm{N}=100^{12}$, Rauch et al: $\mathrm{N}=51^{14}$ ) and developmental delay (DD) (DDD: $\mathrm{N}=1133)^{39}$ (Supplementary Table 2). De novo variants from congenital heart disease ${ }^{26,27}$ and schizophrenia ${ }^{25}$ were also downloaded for additional confirmation of the recurrent mutation rate (Supplementary Tables 5 and 6). Details of the sequencing and de novo calling can be found in the referenced publications.

To ensure uniformity in variant representation and annotation across datasets and with respect to the ExAC reference database ${ }^{40}$, we created a standardized variant representation through a Python implementation of vt normalize $\mathrm{e}^{41}$ and re-annotated all variants using Variant Effect Predictor (VEP) ${ }^{42}$ version 81 with GENCODE v19 on GRCh37. VEP provided the Ensembl Gene IDs, gene symbol, the Ensembl Transcript ID for use in determining canonical transcripts, as well as PolyPhen 2 and SIFT scores. We used the canonical transcript when possible for cases when the variant resided in multiple transcripts, and the most deleterious annotation in cases of multiple canonical transcripts. If no canonical transcript was available, the most deleterious annotation was used. As such, variants in Supplemental Tables 1, 2, and 6 may differ from their respective publications due to standardizing variant representation and annotation.

\section{Determining class 1 or class 2 de novo variants.}

De novo variants were classified as class 1 or class 2 based on their respective absence or presence in ExAC. Presence or absence in ExAC was defined if the variant had the same chromosome, position, reference, and alternate allele in both files. Due to the heterogeneous nature of ExAC, and the different capture arrays used in the original exome sequencing studies incorporated into ExAC, we elected to use all of the variants in ExAC, not just those with a PASS status in the GATK variant calling filter. For insertions/deletions, we took a conservative stance that they must match exactly (i.e., a subset was not sufficient). To illustrate, if a de novo variant on chromosome 5 at position 77242526 has a reference allele of AGATG and a de novo alternate allele where four nucleotides are deleted (AGATG to A), we would not say that variant is present in ExAC if there was another variant at the same genomic position in ExAC where only the first two of these nucleotides are deleted (AGA to A). Lastly, for variants outside of the proportion of the genome covered by ExAC, we considered them to be class 1 de novo variants as expected, none of these variants reside in the coding region (Supplementary Table 34).

\section{Variant calling for transmission and case-control analysis.}

We used the Genome Analysis Toolkit (GATK v3.1-144) to recall a dataset of 22,144 exomes from the Autism Sequencing Consortium (ASC) ${ }^{43} \&$ Simons Simplex Collection (SSC) ${ }^{44}$ sequencing efforts. This call set contained 4319 complete trios (including all those from which 
the published and validated de novo mutations were identified), which we used to evaluate inherited variation, and a published case-control dataset of individuals of Swedish ancestry (404 individuals with ASD and 3564 controls) $)^{5}$. We applied a series of quality control filters on the genotype data, using the genome-wide transmission rate as a guide for filter inclusion/exclusion. More specifically, we calibrated various genotyping filters such that synonymous singleton variants - where the alternative allele was seen in only one parent in the dataset - was transmitted at a rate of $50 \%$, because we expect, as a class, synonymous variants to be transmitted $50 \%$ of the time. As with the ExAC analysis ${ }^{40}$, we found GATK's default Variant Quality Score Recalibration (VQSR) too restrictive due to the bias toward common sites. In order to reduce the number of singleton variants being filtered out, we recalibrated the Variant Quality Score Log Odds (VQSLOD) threshold from -1.49 to -1.754, dropping the singleton synonymous transmission rate from $51.1 \%$ to $49.9998 \%$ (Supplementary Fig. 4). Additional filtering was done at the individual level, in which we required a minimum read depth of 10 and a minimum GQ and PL of 25 for each individual's variant call. We also applied an allele balance filter specific for each of the three genotypes (homozygous reference, heterozygous, homozygous alternate), where allele balance is defined as the number of alternate reads divided by the total number of reads. We required the allele balance for homozygous reference individuals to be less than 0.1 , allele balance for heterozygous individuals to be between 0.3 and 0.7, and the allele balance for homozygous alternate individuals to be greater than 0.9. Calls that did not pass these filters were set to missing. Lastly, for the transmission analysis, we removed variants in which more than $20 \%$ of families failed one of our filters. For the case-control analysis, we removed variants in which more than $5 \%$ of families failed one of our filters.

\section{On the use of the Poisson exact test for comparing rates of de novo variation between two samples.}

As with many other papers ${ }^{6,8,45-47}$, we too were interested in testing whether the rate of a given class of de novo variation was significantly different between our cohorts of individuals with ASD or ID/DD as compared to unaffected ASD siblings. As the number of de novo variants per individual follows a Poisson distribution ${ }^{8}$, we tested $H_{A}: \lambda_{1} \neq \lambda_{2}$ vs. $H_{0}: \lambda_{1}=\lambda_{2}$, where $\lambda_{i}$ is the rate of a given class of de novo variation in group $i$, using the Poisson exact test (also known as the $C$-test) ${ }^{32}$. Note: we could not compare the rates to expectation, because the expectations published in Samocha et al., (2014) are for ALL de novo variants, not just de novo variants present/absent from ExAC. An important consequence of our hypothesis test is that effect sizes are reported as rate ratios (RR), which is simply the quotient of the two rates. While more commonly reported, odds ratios require Bernoulli random variables (e.g., an individual either harbors or does not harbor a de novo variant), and as such, would be incorrect given the hypothesis we are testing. Had we been interested in testing for a significant difference between the proportion of individuals harboring a de novo PTV, then an odds ratio would be appropriate (and Fisher's exact test would suffice in this case). Thus, only in using the Poisson exact test could we reject the null hypothesis that the rate of de novo PTVs is the same between individuals with ASD and their unaffected siblings and find evidence that individuals with ASD have a higher rate of de novo PTVs than their unaffected siblings. The difference between the two tests is a subtle, but important one.

\section{On the use of pLI (probability of loss-of-function intolerance).}


Using the observed and expected number of PTVs per gene in the ExAC dataset, we developed a metric to evaluate a gene's apparent intolerance to such variation ${ }^{24}$. Briefly, the probability of loss-of-function intolerance ( $\mathrm{pLI}$ ) was computed using an EM algorithm that assigned genes to one of three categories: fully tolerant (in which PTVs are presumed neutral and occur, like synonymous variants, at rates proportional to the mutation rate), "recessive-like" (showing PTV depletion similar to known severe autosomal recessive diseases) and "haploinsufficient-like" (showing PTV depletion similar to established severe haploinsufficiencies). pLI is the posterior probability that a gene resides in the last, most loss-of-function intolerant, category. See section 4 of the supplement of Lek, et al. (2016) for more details.

\section{Phenotype Analysis.}

Full-scale deviation IQ scores were measured using several tests including the Differential Ability Scales, the Wechsler Intelligence Scale for Children, and the Wechsler Abbreviated Scale of Intelligence. IQ has previously been associated with de novo PTV rate in the SSC ${ }^{6,15,48}$. In this analysis, we used Poisson regression to estimate the relationship between the rate of each of class 1 and class 2 PTVs and proband full-scale deviation IQ.

\section{Calculating the expected number of class 2 de novo variants in a reference database.}

For a set of $\mathrm{r}$ de novo variants, each with the same allele count, $\mathrm{K}$, in ExAC, we can estimate the number of those variants still observed at least once in a subset of size $\mathrm{n}$ using the hypergeometric distribution. That is to say, how many of those same sites will still be present as standing variation in a down-sampled version of ExAC? Specifically,

$$
\text { expected count }=r(1-\mathrm{P}(\mathrm{k}=0))=\mathrm{r}\left(1-\frac{\left(\begin{array}{l}
\mathrm{K} \\
0
\end{array}\right)\left(\begin{array}{l}
\mathrm{N}-\mathrm{K} \\
\mathrm{n}-0
\end{array}\right)}{\left(\begin{array}{l}
\mathrm{N} \\
\mathrm{n}
\end{array}\right)}\right)=r\left(1-\frac{\left(\begin{array}{c}
\mathrm{N}-\mathrm{K} \\
\mathrm{n}
\end{array}\right)}{\left(\begin{array}{l}
\mathrm{N} \\
\mathrm{n}
\end{array}\right)}\right)
$$

where $\mathrm{k}$ is approximatelyhypergeometric $(\mathrm{N}, \mathrm{K}, \mathrm{n})$, and $\mathrm{N}$ is the number of chromosomes in the current version of ExAC $(\mathrm{N}=121,412)$.

This only holds when each down-sampled set of ExAC preserves the ancestry proportions of the total sample.

\section{Calculating mutation rates for class 1 and class 2 de novo PTVs.}

Samocha et al. calculated per gene mutation rates for ALL synonymous, missense, and PTVs, not for those present/absent in ExAC. If we are interested in comparing the rate of class $1 \mathrm{de}$ novo PTVs to the expected depth-corrected mutation rate for class 1 de novo PTVs, we can roughly calculate it. For a given gene, we can derive the class 1 and class 2 PTV mutation rate by breaking down the overall mutation rate for PTVs, denoted as $\hat{\mu}_{P T V}$, using equation (1)

$$
\hat{\mu}_{P T V}=\hat{\mu}_{\text {class } 1 P T V}+\hat{\mu}_{\text {class } 2 P T V}
$$

In case the logic behind equation 1 isn't completely clear, it may help to point out that the number of class 1 and class 2 PTVs is equal to the total number of PTVs. Now, Samocha et al. provides us with $\hat{\mu}_{P T V}$, so all we need to do is calculate $\hat{\mu}_{\text {class } 1 \text { PTV }}$ and $\hat{\mu}_{\text {class } 2 \text { PTV }}$. Given all of the PTVs in ExAC, and the probability of each trinucleotide-to-trinucleotide mutation, we can calculate $\hat{\mu}_{\text {class } 2 \text { PTV }}$ using equation (2) 
$\hat{\mu}_{\text {class } 2 P T V}=\sum_{P T V_{i}}^{P T V_{n}} \hat{\mu}_{S N P i}$

where $i$ indexes the $n$ PTVs for a given gene present in ExAC, and $\hat{\mu}_{S N P i}$ is the mutation rate of that specific trinucleotide substitution that creates a PTV. With $\hat{\mu}_{\text {class } 2 \text { PTV }}$ calculated, $\hat{\mu}_{\text {class } 1 \text { PTV }}$ follows from equation 1 . However, these per gene $\hat{\mu}_{P T V}$ calculations do not account for sequencing depth. Correcting for depth of sequencing becomes tricky, as the depth of sequencing varies between studies and will not necessarily be the same as the depth of sequencing for ExAC. However, we can roughly approximate the depth-corrected $\hat{\mu}_{\text {class } 2 \text { PTV }}$ for each gene using the following equation under the assumption that the fraction of the raw mutability from class 2 (i.e., $\left.\frac{\widehat{\mu}_{\text {class } 2 P T V}}{\widehat{\mu}_{P T V}}\right)$ is equal to the fraction of the class 2 depth-corrected mutability (i.e., $\left.\frac{\widehat{\mu}_{\text {class } 2 \text { PTV,depth corrected }}}{\widehat{\mu}_{P T V, \text { depth corrected }}}\right)$

$\hat{\mu}_{\text {class } 2 \text { PTV }, \text { depth corrected }}=\hat{\mu}_{P T V, \text { depth corrected }}\left(\frac{\widehat{\mu}_{\text {class } 2 \text { PTV }}}{\widehat{\mu}_{P T V}}\right)$

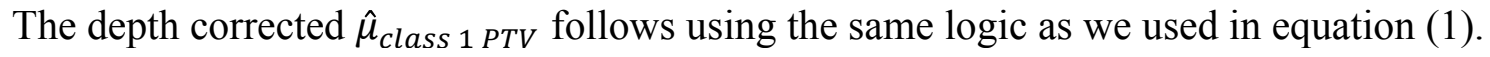

\section{Data availability \& accession codes.}

Data included in this manuscript is deposited in the database of Genotypes and Phenotypes (dbGaP) under accession phs000298.v2.

\section{URLs.}

Exome Aggregation Consortium (ExAC), http://exac.broadinstitute.org/; LOFTEE, https://github.com/konradjk/loftee

\section{Acknowledgements}

We would like to thank all of the members of the ATGU and the Wall Lab for their assistance in this endeavor. We thank the families who took part in the Simons Simplex Collection study and the Simons Variation in Individuals Project, as well as the clinicians who collected data at each of the study sites. The authors would like to thank the Exome Aggregation Consortium and the groups that provided exome variant data for comparison. A full list of contributing groups can be found on the ExAC website (see URLs). We would also like to greatly thank A. Byrnes, R. Fine, D. Fronk, A. Martin, C. Nichols, N. Radd, K. Satterstrom, and E. Wigdor for their insightful contributions. Lastly, we would like to acknowledge G. A. Barnard for inspiring us to write in a more conversational tone, similar to his seminal 1947 paper, Significance Tests For 2x2 Tables. This work was supported by NIH grants U01MH100233, U01MH100209, U01MH100229 and U01MH100239 to the Autism Sequencing Consortium (ASC), and R56 MH097849 and R01 MH097849 to the Population-based Autism Genetics and Environment Study (PAGES). M.J.D., J.A.K., and K.E.S. were supported by grants from the Simons Foundation Autism Research Initiative (SFARI 342292 and a subaward from the Simons Center for the Social Brain at MIT). ML and DGM's work on the ExAC project was funded by U54DK105566 and R01 GM104371 from the National Institutes of Health. KS was funded by T32 HG002295/HG/NHGRI. EBR was funded by National Institutes of Mental Health Grant 1K01MH099286 and NARSAD Young Investigator grant 22379. 


\section{Author Contributions}

JAK and EBR performed the analyses. JAK, DPH, EBR, and MDJ designed the experiment. JAK and KS wrote the code. DPW, EBR, and MJD supervised the research. JAK and MJD wrote the paper. JAK, KES, DPH, SJS, ML, KJK, DGM, and JDB generated data. JAK was responsible for the remainder. All authors revised and approved the final manuscript.

\section{Competing Financial Interests}

The authors declare no competing financial interests. 


\section{Citations}

1. Developmental, D.M.N.S.Y. \& Investigators, P. Prevalence of autism spectrum disorder among children aged 8 years-autism and developmental disabilities monitoring network, 11 sites, United States, 2010. Morbidity and mortality weekly report. Surveillance summaries (Washington, DC: 2002) 63, 1 (2014).

2. Lee, S.H. et al. Genetic relationship between five psychiatric disorders estimated from genome-wide SNPs. Nat Genet 45, 984-94 (2013).

3. Gaugler, T. et al. Most genetic risk for autism resides with common variation. Nat Genet 46, 881-5 (2014).

4. Klei, L. et al. Common genetic variants, acting additively, are a major source of risk for autism. Mol Autism 3, 9 (2012).

5. De Rubeis, S. et al. Synaptic, transcriptional and chromatin genes disrupted in autism. Nature 515, 209-215 (2014).

6. Iossifov, I. et al. The contribution of de novo coding mutations to autism spectrum disorder. Nature 515, 216-221 (2014).

7. Iossifov, I. et al. De novo gene disruptions in children on the autistic spectrum. Neuron 74, 285-99 (2012).

8. Neale, B.M. et al. Patterns and rates of exonic de novo mutations in autism spectrum disorders. Nature 485, 242-5 (2012).

9. O'Roak, B.J. et al. Sporadic autism exomes reveal a highly interconnected protein network of de novo mutations. Nature 485, 246-50 (2012).

10. Sanders, S.J. et al. De novo mutations revealed by whole-exome sequencing are strongly associated with autism. Nature 485, 237-41 (2012).

11. Reichenberg, A. et al. Discontinuity in the genetic and environmental causes of the intellectual disability spectrum. Proceedings of the National Academy of Sciences 113, 1098-1103 (2016).

12. de Ligt, J. et al. Diagnostic exome sequencing in persons with severe intellectual disability. N Engl J Med 367, 1921-9 (2012).

13. Deciphering Developmental Disorders, S. Large-scale discovery of novel genetic causes of developmental disorders. Nature 519, 223-8 (2015).

14. Rauch, A. et al. Range of genetic mutations associated with severe non-syndromic sporadic intellectual disability: an exome sequencing study. Lancet 380, 1674-82 (2012).

15. Robinson, E.B. et al. Autism spectrum disorder severity reflects the average contribution of de novo and familial influences. Proc Natl Acad Sci U S A 111, 15161-5 (2014).

16. Samocha, K.E. et al. A framework for the interpretation of de novo mutation in human disease. Nat Genet 46, 944-50 (2014).

17. Robinson, E.B. et al. Genetic risk for autism spectrum disorders and neuropsychiatric variation in the general population. Nat Genet advance online publication(2016).

18. Bellus, G.A. et al. Achondroplasia is defined by recurrent G380R mutations of FGFR3. Am J Hum Genet 56, 368-73 (1995).

19. Kimura, M. The number of heterozygous nucleotide sites maintained in a finite population due to steady flux of mutations. Genetics 61, 893-903 (1969).

20. Coulondre, C., Miller, J.H., Farabaugh, P.J. \& Gilbert, W. Molecular basis of base substitution hotspots in Escherichia coli. Nature 274, 775-780 (1978). 
21. Haukka, J., Suvisaari, J. \& Lonnqvist, J. Fertility of patients with schizophrenia, their siblings, and the general population: a cohort study from 1950 to 1959 in Finland. Am J Psychiatry 160, 460-3 (2003).

22. Laursen, T.M. \& Munk-Olsen, T. Reproductive patterns in psychotic patients. Schizophr Res 121, 234-40 (2010).

23. Power, R.A., Kyaga, S., Uher, R. \& et al. FEcundity of patients with schizophrenia, autism, bipolar disorder, depression, anorexia nervosa, or substance abuse vs their unaffected siblings. JAMA Psychiatry 70, 22-30 (2013).

24. Lek, M. et al. Analysis of protein-coding genetic variation in 60,706 humans. Nature 536, 285-291 (2016).

25. Fromer, M. et al. De novo mutations in schizophrenia implicate synaptic networks. Nature 506, 179-84 (2014).

26. Homsy, J. et al. De novo mutations in congenital heart disease with neurodevelopmental and other congenital anomalies. Science 350, 1262-1266 (2015).

27. Zaidi, S. et al. De novo mutations in histone-modifying genes in congenital heart disease. Nature 498, 220-3 (2013).

28. Francioli, L.C. et al. Genome-wide patterns and properties of de novo mutations in humans. Nat Genet 47, 822-6 (2015).

29. Genome of the Netherlands, C. Whole-genome sequence variation, population structure and demographic history of the Dutch population. Nat Genet 46, 818-25 (2014).

30. Kong, A. et al. Rate of de novo mutations and the importance of father's age to disease risk. Nature 488, 471-5 (2012).

31. Michaelson, J.J. et al. Whole-genome sequencing in autism identifies hot spots for de novo germline mutation. Cell 151, 1431-42 (2012).

32. Przyborowski, J. \& Wilenski, H. Homogeneity of Results in Testing Samples from Poisson Series: With an Application to Testing Clover Seed for Dodder. Biometrika 31, 313-323 (1940).

33. Picoraro, J. \& Chung, W. Delineation of New Disorders and Phenotypic Expansion of Known Disorders Through Whole Exome Sequencing. Current Genetic Medicine Reports 3, 209-218 (2015).

34. Krumm, N. et al. Excess of rare, inherited truncating mutations in autism. Nat Genet 47, 582-588 (2015).

35. Purcell, S.M. et al. A polygenic burden of rare disruptive mutations in schizophrenia. Nature 506, 185-90 (2014).

36. Singh, T. et al. Rare schizophrenia risk variants are enriched in genes shared with neurodevelopmental disorders. bioRxiv (2016).

37. Akawi, N. et al. Discovery of four recessive developmental disorders using probabilistic genotype and phenotype matching among 4,125 families. Nat Genet 47, 1363-1369 (2015).

38. Richards, S. et al. Standards and guidelines for the interpretation of sequence variants: a joint consensus recommendation of the American College of Medical Genetics and Genomics and the Association for Molecular Pathology. Genet Med 17, 405-24 (2015).

39. Large-scale discovery of novel genetic causes of developmental disorders. Nature 519, 223-8 (2015).

40. Lek, M. et al. Analysis of protein-coding genetic variation in 60,706 humans. bioRxiv (2015). 
41. Tan, A., Abecasis, G.R. \& Kang, H.M. Unified representation of genetic variants. Bioinformatics 31, 2202-4 (2015).

42. McLaren, W. et al. Deriving the consequences of genomic variants with the Ensembl API and SNP Effect Predictor. Bioinformatics 26, 2069-70 (2010).

43. Buxbaum, J.D. et al. The autism sequencing consortium: large-scale, high-throughput sequencing in autism spectrum disorders. Neuron 76, 1052-6 (2012).

44. Fischbach, G.D. \& Lord, C. The Simons Simplex Collection: a resource for identification of autism genetic risk factors. Neuron 68, 192-195 (2010).

45. Ben-David, E. \& Shifman, S. Combined analysis of exome sequencing points toward a major role for transcription regulation during brain development in autism. Mol Psychiatry 18, 1054-6 (2013).

46. Takata, A., Ionita-Laza, I., Gogos, J.A., Xu, B. \& Karayiorgou, M. De Novo Synonymous Mutations in Regulatory Elements Contribute to the Genetic Etiology of Autism and Schizophrenia. Neuron 89, 940-7 (2016).

47. Lelieveld, S.H. et al. Meta-analysis of 2,104 trios provides support for 10 novel candidate genes for intellectual disability. bioRxiv (2016).

48. Sanders, S.J. et al. Insights into Autism Spectrum Disorder Genomic Architecture and Biology from 71 Risk Loci. Neuron 87, 1215-33 (2015). 